Running Head: Attitudes Towards Sunbed Use

A brief report on the role of self-perceived mate value in attitudes towards sunbed use

\author{
Manpal Singh Bhogal*1 \& Alison Leah Owen ${ }^{2}$ \\ ${ }^{1}$ Department of Psychology, Evolution and Human Behaviour Research Group, University of \\ Wolverhampton, WV1 1LY, UK. \\ ${ }^{2}$ Staffordshire Centre for Psychological Research, Psychology Department, School of Life \\ Sciences and Education, Staffordshire University, Stoke-on-Trent, ST4 2DE, UK. \\ *corresponding author: m.s.b2@wlv.ac.uk
}

\title{
Acknowledgments
}

We thank Michaela Hamilton and Azaria Moss for their support in collecting part of the data for this study. 


\begin{abstract}
Prior research suggests that women engage in attractiveness enhancing techniques to increase their mate value. This hypothesis suggests that attractiveness enhancing techniques are driven by evolutionary mechanisms in relation to increasing one's mate value in the mating market. Therefore, people can engage in risky behaviours to achieve this goal, which can involve the use of sunbeds. We explored whether there was a mating motivation in a sample of sunbed users by exploring whether intrasexual competition, mate value, and self-esteem predicted attitudes towards sunbed use $(n=98)$. We find that the only predictor of attitudes towards sunbed use was self-perceived mate value, in that those with higher self-perceived mate value reported negative attitudes towards sunbed use. We discuss these findings in light of evolutionary psychology.
\end{abstract}

Keywords: sunbed use, mate value, intrasexual competition, self-esteem, attractiveness 


\title{
A brief report on the role of self-perceived mate value in attitudes towards
}

\author{
sunbed use
}

\section{Sunbed use}

The aim of this project was to further explore the evolutionary determinants of attitudes towards sunbed use amongst sunbed users. Evolutionary explanations have been proposed to explain the drivers of attractiveness enhancing, risky health behaviour, suggesting people engage in risky health behaviour to increase attractiveness, and to subsequently attract mates. We aimed to explore whether there is a role of intrasexual competition, self-perceived mate value (which to our knowledge have not been explored in the literature), and self-esteem in attitudes towards sunbed use.

The negative consequences deriving from the use of attractiveness enhancement practices has been well documented in the literature. Health Psychologists and healthcare practitioners have created education programmes designed to make people aware of the risks associated with risky practices. Sunbed use is one of those risky behaviours, which can be practiced to enhance one's attractiveness (Schneider et al. 2013). Research suggests that women (see Nasser, 1988) typically engage in the use of attractiveness enhancing risky practices, such as sunbed use, even when they are aware of the risks associated with UV exposure (Olson et al. 2008), and this is usually driven by the goal of enhancing one's physical attractiveness (Saad \& Peng, 2006). In addition, researchers suggest that this increase in attractiveness can lead to higher confidence and self-esteem, suggesting selfesteem plays a role in tanning behaviour (Noar et al. 2014). Further research is needed to explore the determinants of sunbed use, considering skin cancer rates have dramatically increased over recent years, with melanoma skin cancer incidence rates having increased by half $(50 \%)$ in the UK over the past decade, and with incidence rates for melanoma skin 
cancer projected to rise by $7 \%$ in the UK between 2014 and 2035 (Cancer Research UK, 2018).

The World Health Organization (2018) reports that regular use of sunbeds can significantly increase a person's chances of getting skin cancer. Sunbed use is a serious issue in the UK. For example, in a meta-analysis looking at the impact of a UV exposure intervention, Williams, Grogan, Clark-Carter and Buckley (2013) found that almost a fifth (18.6\%) of the participants in their study had used a sunbed at least once in the past month, with the majority of participants agreeing that a tan looked good (80\%), and that tanned people look healthy $(71.4 \%)$. The findings differed dramatically to their study with men (Wiliams, Grogan, Clark-Carter \& Buckley, 2016), with just 7\% of the participants reporting using a sunbed in the past month. This forms a rationale for exploring sunbed use amongst women.

\section{Evolutionary drivers of sunbed use}

Due to lack of research exploring evolutionary explanations for sunbed use, we aim to explore whether people engage in risky, attractiveness enhancing practices due to evolutionary drivers. In support, research suggests that women engage in tanning behaviour due to partner preferences for tanned skin (Mosher \& Danoff-Burg, 2005). Consistent with evolutionary psychology, due to the fact that men value attractiveness in a mate far more than women, women do compete with one another on attractiveness, which may lead to attractiveness enhancing practices (Bhogal, Galbraith \& Manktelow, 2018; Keys \& Bhogal, 2018), hence why the sample for our study was female. This competition can be explained by intra-sexual competition, which is defined as members of the same sex competing on characteristics which are attractive to the opposite sex (Keys \& Bhogal, 2018), in this case, competing to be attractive through the use of sunbeds. 
Supporting the rationale for exploring tanning behaviour amongst women only, Banerjee, Campo and Greene (2008) found that when participants were presented of models with a varying tan, men rated models with a darker tan as more attractive than light or medium tans. Furthermore, there was no difference in women's attractiveness ratings when looking at male models with a varying tan. In addition, women in the sample stated that they engaged in tanning behaviour and general health choices were driven by appearing attractive to potential mates, believing a tan would increase their physical attractiveness. In addition, men rate tanned women as healthier and more attractive compared to non-tanned women (Bannerjee et al. 2008). This preference for attractive women is driven by evolutionary pressures to find good genetic quality mates in order to contribute to the production of highquality offspring (Buss, 1994). These evolutionary pressures can stem from intrasexual competition to be attractive.

As a result of existing research, we suggest that attitudes towards sunbed use may be motivated by mating goals (Pettijohn, Pettijohn, \& Gilbert, 2011). Although research into sunbed use has focused on health-related models, research does suggest that attractivenessenhancement, specifically, social-self-representation largely predicts engagement in risky health practices (Leary \& Jones, 1993). For example, Leary and Jones (1993) found that women who engaged in sunbed use were well aware of the dangerous risks posed, however, the immediate benefits associated with increased attractiveness, outweighed their long-term health concerns, signifying the need to adopt an evolutionary model to understand sunbed use. As outlined previously, sunbed use can be a dangerous, attractiveness-enhancing behaviour. Research has adopted health-belief models to explore this behaviour, however social and evolutionary consequences and mechanisms must also be explored. Hill and Durante (2011) aimed to explore whether activating mating goals (through priming, showing women attractive same-sex and opposite sex faces) increase womens' willingness to engage 
in tanning behaviour. Through two experiments, they primed participants with courtship stimuli and neutral stimuli, finding women were more willing to take diet pills and engage in tanning when mating goals were activated (when intrasexual competition was high), suggesting there is an evolutionary explanation for engaging in attractiveness enhancing health behaviours. Furthermore, they found that when mating goals were activated, women were less likely to believe they were susceptible to the health risks posed by diet pills and tanning, signifying the strong role mate attraction/intra-sexual competition plays in the use of attractiveness enhancing behaviours. Hill and Durante (2011) explored hypothetical intention with facial images, whereas we aim to further explore beliefs and attitudes towards sunbed use, as beliefs predict actual health behaviour (see Jackson \& Aiken, 2000).

Taken as a whole, these variables suggest that interpersonal, and internal factors may drive the use of sunbeds. As a result of the aforementioned literature, we hypothesised the following:

H1: Self-perceived mate value will be negatively related to attitudes towards sunbed use H2: Intrasexual competition will be positively related to attitudes towards sunbed use. H3: Self-esteem will be negatively related to attitudes towards sunbed use.

\section{Method}

\section{Design and participants}

A cross-sectional correlational design was adopted to explore whether intrasexual competition, self-perceived mate value, and self-esteem predicted attitudes towards sunbed use. The sample comprised of 98 heterosexual women from the general public in the UK, who were sunbed users, recruited via Qualtrics $($ Mage $=28.61$ years old, $S D=11.77)$. Women were recruited due to the theoretical foundation of the study, consistent with previous 
research (e.g. Stapleton et al. 2016). Furthermore, a meta-analysis revealed that women are far more likely to use sunbeds compared to men (Wehner et al. 2014).

To guide our anticipated sample size, an a-priori power analysis was conducted using G*Power (Faul, Erdfelder, Buchner \& Lang, 2009). To achieve $80 \%$ power (medium effect size of .15 with 3 predictors, and an alpha level of .05 - all for multiple regression), G*Power recommended 77 participants, which we surpassed.

\section{Materials}

Attitudes towards sunbed use

The 13-item Attitudes towards sunbed use scale (Owen, Grogan, Clark-Carter, Buckley, 2013) was used to explore participants' attitudes towards sunbed use, with each item measured on a 1 (strongly agree) to 5 (strongly disagree) Likert scale. An example (one which is reverse scored) from the scale is 'Sun and UV light damage cause wrinkles'. For all reliability analyses, we used McDonalds Omega, as it provides a more robust measure of scale reliability (Dunn, Baguley, \& Brunsden, 2014). The scale was reliable in our sample $($ Omega $=.74)$.

\section{Mate Value}

The 17-item Mate Value Inventory Short Form (MVI-7; Kirsner et al., 2003) was used to measure the participants' self-perceived mate value. The MVI-7 lists 17 characteristics which are scored on a 7-point Likert scale ranging from "-3 = Extremely low on this characteristic" to " $+3=$ Extremely high on this characteristic". High scores reflect high mate value. Examples items include "attractive face" and "financially secure". The scale was reliable in our sample $($ Omega $=.78)$. 


\section{Intrasexual competition}

The 12-item intrasexual competition scale (Buunk \& Fisher, 2009) was used to measure intrasexual competition. The items are measured via a 1 (not at all applicable) to 7 (completely applicable), with higher scores reflecting high intrasexual competition. The male version was presented to men, and the female version was presented to women (the items are the same, but the gendered pronouns are sex-specific. For example, the item 'I always want to beat other women.' Was presented to women, and the item 'I always want to beat other men' was presented to men. The scale was reliable in our sample $($ Omega $=.90)$.

\section{Self-esteem}

The 10-tem Self-esteem (Rosenberg, 1965) scale was used to measure participants' selfesteem. The scale measures an individual's self-esteem, with high values reflecting high selfesteem. The scale includes items such as 'On the whole, I am satisfied with myself'. The scale was reliable in our sample $($ Omega $=.93)$.

\section{Procedure}

Once participants provided informed consent, they were asked to provide demographic details and complete the questionnaires outlined above. Finally, participants were fully debriefed. Data were collected anonymously, online, using Qualtrics.

\section{Results}

Descriptive statistics are presented in Table 1. Bivariate Pearson's correlations between all variables are presented in Table 2. Table 2 shows that there was a significant, negative relationship between self-perceived mate value and attitudes towards sunbed use, in 
that higher mate value was associated with lower (more negative) attitudes towards sunbed use.

\section{Multiple Regression}

Multiple regression was conducted to predict attitudes towards sunbed use (see Table 3 for statistics derived from the analysis) from self-perceived mate value, self-esteem, and intrasexual competition. Preliminary analyses were conducted to ensure no violation of the assumptions of normality, linearity, multicollinearity, homoscedasticity, and independent errors. The model explained $9.3 \%$ of the variance in attitudes towards sunbed use, $F(3,94)=$ 3.22 (adjusted $\left.R^{2}=.064\right), p=.026$. Self-perceived mate value was a significant, negative predictor of attitudes towards sunbed use, as opposed to self-esteem and intrasexual competition were non-significant predictors of attitudes towards sunbed use. As a result, all hypotheses were refuted, apart from $\mathrm{H} 2$, which was fully supported.

(Insert Tables 1, 2, and 3 here)

\section{Discussion}

The aim of this study was to further explore evolutionary predictors of attitudes towards sunbed use in a sample of sunbed users. Our findings provide partial support support for our hypotheses, whereby self-esteem, and intrasexual competition did not predict attitides towards sunbed use. However, we did find that self-perceived mate value was a strong negative predictor of attitudes towards sunbed use.

This key finding that mate value predicts attitudes towards sunbed use can be interpreted under the lens of evolutionary theory. Previous research suggests that people use sunbeds in order to increase their attractiveness, as tanned skin has been found to be attractive 
in mate choice contexts (Mosher \& Danoff-Burg, 2005). Furthermore, due to the fact attractiveness is a sign of genetic quality, it has evolved as a sexually selectable trait, meaning it is highly valued in mate choice (Bhogal et al. 2019).

This negative relationship could reflect that those who have higher mate value do not necessarily need to use sunbeds frequently to increase their attractiveness, as they already possess the traits and qualities which add to their overall mate value. However, those with lower mate value may have more positive attitudes towards sunbed use due to the fact that using sunbeds can increase one's attractiveness. To our knowledge, this is the first empirical study to report an association between mate value and sunbed use in particular, meaning future research could further unpack this association by looking at additional evolutionary predictors such as mate value discrepancy and the actual use of sunbeds. If having tanned skin is attractive, then mate value discrepancies between one's mate value and their partner could predict whether people engage in risky attractiveness enhancing behaviours to 'keep' a romantic partner. In support, mate value discrepancy has been applied to several areas such as cyberpsychology (Bhogal \& Howman, 2019).

There are limitations to consider with this study. First, we did not gather data regarding participants' long-term usage of sunbeds, which could have influenced the findings. Perhaps more frequent use of sunbeds over a longer period of time could have influenced the results. As a result, future research should gather past sunbed use in terms of frequency. Second, men could also be included in the sample, as research does suggest that a small percentage of men do engage in sunbed use (Wiliams et al. 2016). However, our focus was on women, due to exploring sunbed use as an attractiveness enhancing method from an evolutionary perspective. Third, the design of this study was cross-sectional, meaning causality cannot be determined. Last, future research could explore the role of body esteem, 
and whether intrasexual competition moderates the relationship between body esteem and attitudes towards sunbed use.

In summary, we provide preliminary evidence that mate value predicts attitudes towards sunbed use in a sample of sunbed users. Future research could further unpack this association and delve further into how attitudes and the use of sunbeds are driven by evolutionary factors. This study is novel, in that it provides preliminary evidence based on theory, that shows evolutionary predictors play a role in tanning behaviour.

\section{Compliance with Ethical Standards}

On behalf of all authors, the corresponding author states that there is no conflict of interest. This research involved collecting data from human participants. Informed consent was taken from all participants who took part in this study. All procedures performed in studies involving human participants were in accordance with the ethical standards of the institutional and/or national research committee and with the 1964 Helsinki declaration and its later amendments or comparable ethical standards. 


\section{References}

Banerjee, S., Campo, S., \& Greene, K. (2008). Fact or wishful thinking? Biased expectations in 'I Think I look better when I'm tanned. American Journal of Health Behavior, 32, 243-252.

Bhogal, M. S., Galbraith, N., \& Manktelow, K. (2019). A research note on the influence of relationship length and sex on preferences for altruistic and cooperative mates. Psychological Reports, 122(2), 550-517. DOI:10.1177/0033294118764640

Bhogal, M. S., \& Howman, J. M. (2019). Mate value discrepancy and attachment anxiety predict the perpetration of dating abuse. Evolutionary Psychological Science, 5(1), 113120.

Buss, D. M. (1994). The evolution of desire: Strategies of human mating. New York: Basic Books.

Buunk, A. P., \& Fisher, M. (2009). Individual differences in intra-sexual competition. Journal of Evolutionary Psychology, 7(1), 37-48.

Cancer Research UK. (2018). https://www.cancerresearchuk.org/health-professional/cancer$\underline{\text { statistics-for-the-uk }}$

Dunn, T. J., Baguley, T., \& Brunsden, V. (2014). From alpha to omega: a practical solution to the pervasive problem of internal consistency estimation. British Journal of Psychology, 105(3), 399-412.

Faul, F., Erdfelder, E., Buchner, A., \& Lang, A.G. (2009). Statistical power analyses using G*Power 3.1: Tests for correlation and regression analyses. Behavior Research Methods, 41, 1149-1160.

Hill, S. E., \& Durante, K. M. (2011). Courtship, competition, and the pursuit of attractiveness: mating goals facilitate health-related risk taking and strategic risk suppression in women. Personality and Social Psychology Bulletin, 37, 383-394. 
Jackson, K. M., \& Aiken, L. S. (2000). A psychosocial model of sun protection and sunbathing in young women: The impact of health beliefs, attitudes, norms, and selfefficacy for sun protection. Health Psychology, 19(5), 469-478.

Keys, E., \& Bhogal, M. S. (2018). Mean girls: provocative clothing leads to intra-sexual competition between females. Current Psychology, 37(3), 543-551.

Kirsner, B. R., Figueredo, A. J., \& Jacobs, W. J. (2003). Self, friends, and lovers: structural relations among beck depression inventory scores and perceived mate values. Journal of Affective Disorders, 75, 131-148.

Leary, M. R., \& Jones, J. L. (1993). The social psychology of tanning and sunscreen use: self-presentational motives as a predictor of health risk. Journal of Applied Social Psychology, 23(17), 1390-1406.

Mosher, C. E., \& Danoff-Burg. (2005). Social predictors of sunscreen and self-tanning product use. Journal of American College Health, 54, 166-168.

Nasser, M. (1988). Culture and weight consciousness. Journal of Psychosomatic Research, 32(6), 573-577.

Noar, S. M., Myrick, J. G., Morales-Pico, B., \& Thomas, N. E. (2014). Development and validation of the comprehensive indoor tanning expectations scale. JAMA Dermotology, 150(5), 512-521.

Olson, A. L., Gafney, C. A., Starr, P., \& Dietrich, A. J. (2008). The impact of an appearancebased educational intervention on adolescent intention to use sunscreen. Health Education Research, 23(5), 763-769. 
Owen, A. L., Grogan, S., Clark-Carter, D., \& Buckley, E. (2013). British adolescents' sun protection and UV exposure awareness. British Journal of School Nursing, 8(9), 436441.

Owen, A. L., Grogan, S., Clark-Carter, D., \& Buckley, E. (2018) The impact of an appearance-focused facial-ageing intervention on adolescents' attitudes toward sun protection. British Journal of School Nursing, 13(9), 436-444.

Pettijohn, T. F., Pettijohn, T. F., \& Gilbert, A. G. (2011). Romantic relationship status and gender differences in sun tanning attitudes and behaviors of U.S. college students, Psychology, 2(2), 71-77.

Saad, G., \& Peng, A. (2006). Applying Darwinian principles in designing effective intervention strategies: The case of sun tanning, Psychology and Marketing, 23(7), 617638.

Schneider, S., Diehl, K., Dipl-Soz, Bock, C., Schluter, M., Breitbart, E. W., Volkmer, B., \& Greinert, R. (2013). Sunbed use, user characteristics, and motivations for tanning. Results from the German population-based SUN-study 2012. JAMA Dermatology, 149(1), 43-49.

Stapleton, J. L., Manne, S. L., Greene, K., Darabos, K., Carpenter, A., Hudson, S. V., \& Coups, E. J. (2016). Sociocultural experiences, body image, and indoor tanning among young adult women. Journal of Health Psychology, 1-9.

Williams, A. L., Grogan, S., Clark-Carter, D., \& Buckley, E. (2013). Appearance-based interventions to reduce ultraviolet exposure and/or increase sun protection intentions and behaviours: a systematic review and meta-analyses. British Journal of Health Psychology, 18(1), 182-217.

Word Health Organisation. (2018).www.who.int/gho/publications/world_health_statistics/en 
Table 1: Descriptive statistics relating to all key variables

Variable Total mean $(S D)$

Attitudes towards sunbed use

$23.32(5.75)$

Self-perceived mate value

$83.81(11.62)$

Self-esteem

$21.40(4.39)$

Intrasexual competition

$28.01(13.37)$ 
Table 2: Bivariate correlations between attitudes towards sunbed use, self-perceived mate value, self-esteem, and intrasexual competition.

\begin{tabular}{lcccc}
\hline & 1 & 2 & 3 & 4 \\
\hline 1. Sunbed use & - & $-.28^{* *}$ & .14 & -.12 \\
2. Mate value & - & - & $-.32 *$ & -.04 \\
3. self-esteem & - & - & - & .19 \\
4. Intrasexual competition & - & - & - & - \\
\hline
\end{tabular}

Note: $p<.01^{* *}, p<.05^{*}$ 
Table 3: Results of the multiple regression model predicting attitudes towards sunbed use from self-perceived mate value, self-esteem, and intrasexual competition.

\begin{tabular}{|c|c|c|c|c|c|c|}
\hline Variable & $\beta$ & $p$ & $t$ & CI (95\%) & Tolerance & VIF \\
\hline Mate value & -.275 & .007 & -2.74 & $\begin{array}{lll}.24 & -.04\end{array}$ & .958 & 1.044 \\
\hline Self-esteem & .039 & .701 & .39 & $-.21 \quad .31$ & .943 & 1.060 \\
\hline Intrasexual & -.127 & .203 & -1.28 & $-.14 \quad .03$ & .983 & 1.017 \\
\hline competition & & & & & & \\
\hline
\end{tabular}

